\title{
Neutrino Velocity: Results and prospects of experiments at beamlines other than CNGS
}

\author{
P. Adamson ${ }^{\mathrm{a}}$ \\ ${ }^{a}$ Fermi National Accelerator Laboratory, PO Box 500, Batavia, IL 60510, USA
}

\begin{abstract}
In response to the 2011 report of superluminal neutrinos made by the OPERA collaboration, both MINOS and T2K developed plans to upgrade their timing systems to be able to measure neutrino time-of-flight with increased accuracy. In addition, MINOS has undertaken an analysis of the data taken with their old timing system, substantially improving the accuracy of their 2007 measurement, and deriving a result fully consistent with neutrinos travelling at the speed of light.
\end{abstract}

Keywords: neutrino, velocity, time-of-flight, MINOS, T2K

\section{History of neutrino velocity measurements}

The earliest measurements of the speed of the neutrino were made in the 1970s, with the Fermilab Main Ring narrow-band neutrino beam. By comparing the arrival times of neutrinos and muons, a limit on the fractional difference between the neutrino speed and the speed of light $|v / c-1|<4 \times 10^{-5}$ is obtained at the $95 \%$ confidence level [1] with neutrinos of energies 30-200 $\mathrm{GeV}$ over a baseline of about $900 \mathrm{~m}$.

The observation, by the Kamiokande [2], IMB [3] and Baksan [4] experiments of 24 neutrino events from supernova SN1987A within a 30s period allows one to place a tighter constraint on the velocity of these neutrinos. The first light from SN1987A was observed some 3 hours after the neutrino pulse, but as nobody was watching the Large Magellanic Cloud in the hours prior to this observation, this is only a limit. Combining this observation with supernova models, Longo [5] derives a limit $|v / c-1|<2 \times 10^{-9}$ for the speed of neutrinos with energies $\sim 10-40 \mathrm{GeV}$, over a baseline of 168,000 light years.

Tighter constraints are derived by Cohen and Glashow [6], arguing that superluminal neutrinos must produce $\mathrm{e}^{+} / \mathrm{e}^{-}$pairs via brehmsstrahlung, and by Davies and Moss [7] with arguments based on nucleosysthe- sis in the early universe and acoustic oscillations in the cosmic microwave background, but these are not direct measurements of the neutrino velocity.

\section{Measuring Neutrino Velocity in a Long Baseline Experiment}

In the conventional neutrino beam facilities under discussion here, neutrinos are produced in the decay of pions and kaons formed by the interaction of a primary proton beam with a production target. The neutrinos therefore inherit the time structure of the parent proton beam, which is determined by the accelerator that produces it. It will be apparent that having the proton beam bunched in a time structure which is resolvable by the single event timing resolution of your detector will be a distinct advantage to this enterprise. There is a small additional smearing that arises from the spread in pion and kaon energies, and from the distance travelled before they decay, but this effect is of order $100 \mathrm{ps}$ and so can be ignored.

Measuring the neutrino velocity is conceptually straightforward. One requires two detectors separated by some well-measured distance, and accurate timing at each detector. In the case of the CNGS experiments - 
OPERA, ICARUS, LVD and Borexino, a beam current transformer measuring the parent proton beam is used as the near site detector. MINOS and T2K are able to chose between obtaining near site timing from the parent protons or from their respective near neutrino detectors.

\subsection{Baseline determination}

The distance between survey markers on the surface at the near and far sites may be measured at the centimeter level with GPS geodesy [8]. Measuring the relative location of the shallow underground near detector to an accuracy better than this is typically straightforward. The dominant error on the baseline measurement comes from the transport of the survey down a long access tunnel or mine shaft between the surface and the deep underground far detector, and in the case of all current experiments has an uncertainty of tens of centimeters.

\subsection{Time measurement and time transfer}

The measurement of the precise time of a neutrino event is not important for oscillation physics. Experiments use the time of arrival of a neutrino at their far detector to reject cosmic background, but as beam spills are several microseconds in length, having the event time accurate to a microsecond is perfectly satisfactory. By contrast, the relative timing between electronics channels has always been important, and is used in MINOS to distinguish upward and downward-going muons for the atmospheric analysis.

Precision time transfer over long distances is routinely performed by time labs around the world, such as NIST and USNO in the US and NICT and NMIJ in Japan. Each lab operates an ensemble of clocks to produce their own representation of UTC and measures their clocks against other labs and against the BIPM, who calculates a global average UTC.

These laboratories compare timescales by one of three satellite-based methods. Two methods exploit the freely-available GPS satellite system. GPS Common View (CV) requires the simultaneous observation of signals from one or more GPS satellites at both locations. Given that one knows the location of the GPS receivers, observation of the same event in both places determines the relative offset between each set of clocks.

The second GPS method, Precise Point Positioning (PPP) arose in the geodetic community and has been adopted for precise timing in recent years. One records observations of all in-view satellites with a dualfrequency geodetic GPS receiver. Offline, measurements of the satellites by an ensemble of reference stations at known locations are used to calculate precise satellite orbits, which in turn are combined with the records from the GPS receiver in question to determine a solution for position and time.

The third method, two-way satellite time and frequency transfer (TWSTFT), sends signals between the two sites via a dedicated geostationary satellite. Under the assumption that the time for the signal to travel from $A$ to $B$ and B to A is the same, the times between transmitted and received pulses at each site can be compared to determine the relative clock offset. Because it requires both dedicated satellite time and costly hardware at each end of the link, TWSTFT is considerably more expensive than the GPS techniques.

With careful use, all these techniques can achieve accuracies at the level of a nanosecond or two [9].

\section{T2K plans}

The baseline of the T2K experiment [10], at $295 \mathrm{~km}$ is close to a factor of 2.5 shorter than that of either MINOS or CNGS, so it stands disadvantaged, ceteris paribus, by that factor in the determination of neutrino velocity. It does offer the lowest energy beam, with a mean energy of around $600 \mathrm{MeV}$. The T2K proton bunches are roughly $40 \mathrm{~ns}$ in length and separated by about $580 \mathrm{~ns}$, and are easily resolvable by the detectors.

As of June 2012, GPS timestamps are applied to events recorded at the near (JPARC) and far (Super-Kamiokande) sites based on traditional singlefrequency GPS timing receivers, and achieving a precision of around $30 \mathrm{~ns}$ at the $95 \%$ level. A programme of timing upgrades is in progress, both to install more accurate equipment and to better measure internal delays. T2K expects to have modern dual-frequency GPS receivers operating in common view mode beginning in the spring of 2013, and plans regular cross-calibrations by carrying atomic clocks and GPS receivers between the sites. They expect to achieve a single-event timestamp precision better than $10 \mathrm{~ns}$, which will be dominated by the length of the proton bunch.

Final calculations of the expected timing accuracy have not been made, but it is the opinion of the present author that an accuracy of a small number of nanoseconds is achievable. 


\section{MINOS plans}

Following the OPERA announcement [11] in September 2011, MINOS [13] began a collaboration with the precise time experts of the National Institute of Standards and Technology (NIST) and the United States Naval Observatory (USNO). A precise timekeeping system has been designed and installed, and was operated during the final two months of the MINOS run, before the year-long accelerator shutdown that began in May 2012. The analysis of this data is ongoing, and is expected to be completed later in 2012 .

The guiding principle of the new timing system is not to disturb the existing electronics, but to measure its behaviour against a good time reference, and then correct the data offline. To that end, an HP5071A cesium atomic clock is placed by each detector, and detector timing signals are measured against it with Agilent 53230A time interval counters (TICs). The clock's $10 \mathrm{MHz}$ and pulse per second (PPS) signals are transported to the surface over single-mode optic fiber via two-way time transfer with Linear Photonics TIMELINK and more TICs. This use of two-way time transfer renders the measurement immune to the variation of the fiber length with temperature; over the course of the experimental run a 2 ns effect at the Far Detector was observed.

At the surface, the transported clock signals are measured by multiple dual-frequency GPS receivers in custom chassis. The receivers are NovAtel OEMV and OEM6, the antennas are NovAtel 702-GG and 703-GG, and the antenna cables are Andrew FSJ-1, made as short as possible and temperature-annealed for reduced temperature sensitivity. The residual effect due to temperature sensitivity of the antenna cable is estimated to be less than $100 \mathrm{ps}$.

Observations of code and carrier phase on both L1 and L2 frequencies are recorded for each GPS receiver in RINEX and CGGTTS files; these are processed both with PPP and with the common-view technique.

There are two permanent GPS receivers at each detector site (MINOS has three such sites - the MI60 accelerator building, containing a resistive wall current monitor measuring the proton waveform, the near detector (ND) building above the MINOS near detector, and the far detector (FD) in Soudan, MN. In addition, two additional GPS receivers, complete with antenna cables and antennas, are transported between sites in order to achieve a relative calibration of the internal delay of the GPS receivers and to verify that this has not drifted over time.

Initial indications show a stability and accuracy of better than $0.5 \mathrm{~ns}$ on the link between the MINOS near and far detectors. The MINOS bunches have less than $1 \mathrm{~ns} \sigma$, and are seperated by $18.8 \mathrm{~ns}$, and the single-event timing resolution in each detector is between 1 and $2 \mathrm{~ns}$.

\section{MINOS retrospective results}

In 2007, MINOS [12] measured neutrinos arriving at the far detector

$$
\delta=-126 \pm 32 \text { (stat) } \pm 64 \text { (syst.) ns }
$$

after the lightspeed expectation-a central value that is superluminal by 1.8 standard deviations. The measurement presented here results from an additional five years of running, increasing the number of recorded neutrino events by a factor of more than 8 . In addition, substantial improvements in the systematic uncertainties and understanding of the quirks of the old timing system have been made.

\subsection{Improved Systematics}

The largest source of uncertainty in the 2007 result was the length of the optical fibers carrying the GPS signals from the antenna to the GPS receiver located underground by the detector. The uncertainty arose from different optical instruments yielding different measurements of the length of this fiber. In early 2012, these delays were remeasured electrically, including the RFoptical converters in the measurement. Frequency domain measurements with a network analyzer and time domain measurements with a pulse generator and oscilloscope were consistent at the $1 \mathrm{~ns}$ level. Table 1 shows the resultant improvement in this measurement.

\begin{tabular}{|l|c|c|}
\hline GPS antenna fiber & 2007 & 2012 \\
\hline Near Detector & $1275 \pm 29 \mathrm{~ns}$ & $1309 \pm 1 \mathrm{~ns}$ \\
Far Detector & $5140 \pm 46 \mathrm{~ns}$ & $5098 \pm 2 \mathrm{~ns}$ \\
\hline
\end{tabular}

Table 1: 2007 and 2012 determinations of the length of the GPS antenna fiber for the 2005-2006 run period. In 2012, the uncertainty on the FD measurement is larger than the ND because it incorporates the measured length of a small section of fiber destroyed by the 2011 fire in the Soudan mine shaft.

The other large uncertainty in 2007 was a 32 ns uncertainty on the latency of the near detector readout electronics. MINOS replaced the 2007 dead reckoning based on bench measurements of the electronics with a measurement of the total relative latency of the near and far detectors. Two identical, portable "auxiliary detectors" were constructed consisting of a pair of $0.5 \mathrm{~m} \times 0.5 \mathrm{~m}$ planes of MINOS-style scintillator strips 
read out by standard MINOS Hamamatsu M16 multianode photomultipliers. The coincidence of the two planes was timed with respect to a detector timing signal with a Brilliant Instrument BI200 time interval analyzer. Both detectors were operated at the Near Detector, then one was transported to the Far Detector. Measuring the relative time of muon tracks in the MINOS detector and auxiliary detector allowed the determination of the relative latency of the Near and Far detectors with 1 ns accuracy.

\subsection{Old timing system quirks}

Using the upgraded timing equipment, MINOS was able to determine a number of unexpected quirks in the event timing. Absolute time, in the old system, was derived from a Truetime XL-AK GPS timing receiver. This receiver has a random offset with respect to GPS time that appears stable over time, but changes each time it is power cycled. This behaviour, under test conditions, is shown in figure 1. Our measurements indicate that we expect this GPS offset to have an RMS of about $60 \mathrm{~ns}$ (see figure 2.)

Over the 8 years of MINOS running, one or other GPS receiver was power-cycled on 29 occasions. Each period between power cycles was considered an independent measurement of the neutrino time of flight, with a random offset as described above. Considering only periods that contain at least 100 events at the far detector, in order to have a reasonable quality of fit, the data are divided into 19 datasets.

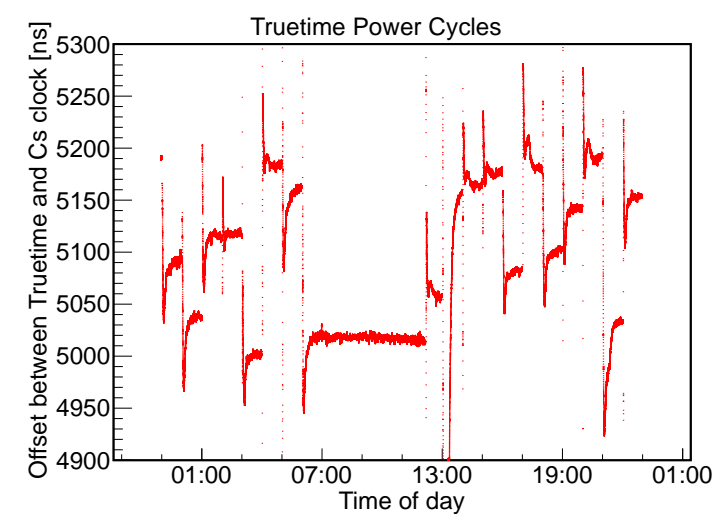

Figure 1: Offset between old GPS timing receiver and a good cesium atomic clock. Step changes occur when the GPS receiver is powercycled. Power cycles occurred automatically on the hour for most of the day. Offset from zero here is arbitrary.

\subsection{Two analysis methods}

The data were analyzed in two different ways. The first method, known as the "full spill approach" closely

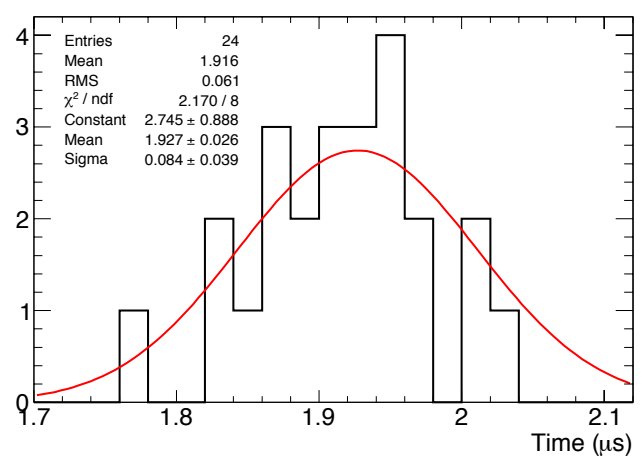

Figure 2: Distribution of offsets of old GPS timing receiver with respect to GPS time. Offset from zero here is arbitrary.

follows the 2007 analysis [12]. The data at the near detector is used to form a high statistics prediction for the time distribution at the FD, and an unbinned maximum likelihood fit is performed to determine the time offset.

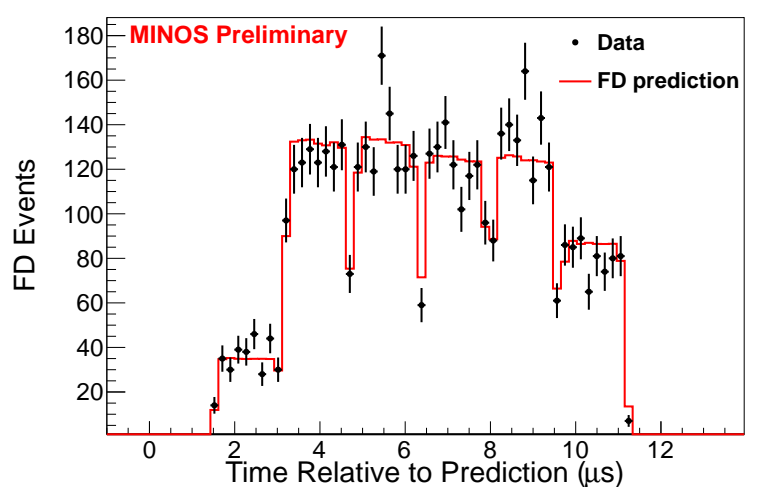

Figure 3: Fit to the far detector dataset in the "full spill" method.

The second method is known as the "wrapped batch" method. Exploiting the fact that the beam from the Main Injector is structured in six "Booster batches", each consisting of 81 consecutive $53 \mathrm{MHz}$ RF bunches, separated by typically a five bunch $(\sim 100 \mathrm{~ns})$ gap, the data from each batch are overlaid to obtain a higher precision measurement of the location in time of the gap between batches.

The data from the ND and FD are fit separately to determine the location of the gap, then the fit results subtracted to obtain a measurement of the time of flight. This method is illustrated in figure 4.

The results from these two methods, for the 19 time periods between GPS power cycles, are shown in figure 5. One observes good agreement for both methods 


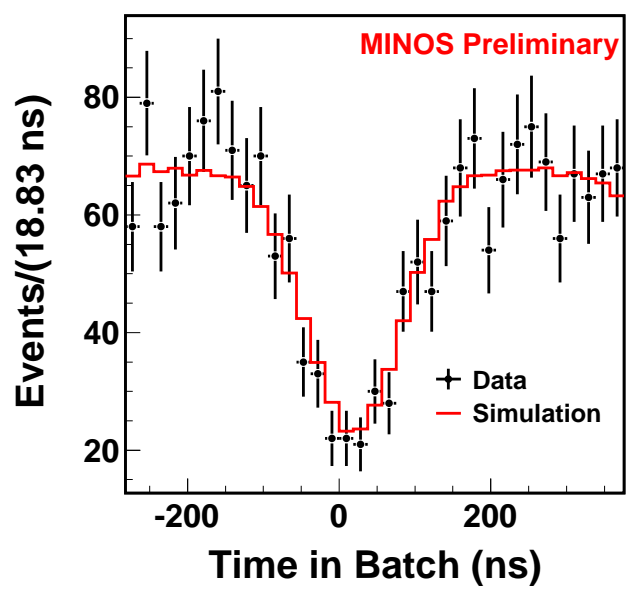

Figure 4: Fit to the far detector dataset in the "wrapped batch" method.

for all datasets. Combining the results produces a measurement of the discrepancy of the time of flight with respect to the speed of light of

$$
\delta=-18 \pm 11 \text { (stat) } \pm 29 \text { (syst.) ns }
$$

for the full spill method, and

$$
\delta=-11 \pm 11 \text { (stat) } \pm 29 \text { (syst.) ns }
$$

for the wrapped batch method.

The systematic error is dominated by the $21 \mathrm{~ns}$ uncertainty on the relative internal delay of the GPS receivers at the ND and FD. In principle, one could measure this rather better with some dedicated time and effort, but in practice the new, modern GPS system will render the idiosyncrasies of the old system of mere historical interest.

Combining these leads to a measurement of the fractional difference between the neutrino velocity and the speed of light

$$
\left(\frac{v}{c}-1\right)=0.6 \pm 1.3 \times 10^{-5}(68 \% \text { C.L. })
$$

\section{Conclusions}

MINOS has updated its 2007 analysis with a factor of eight more data and a better understanding of the systematic errors, and finds no evidence for superluminal neutrinos. MINOS has installed an updated timing system, and with two months of data from spring 2012, expects to measure the neutrino time of flight with a small

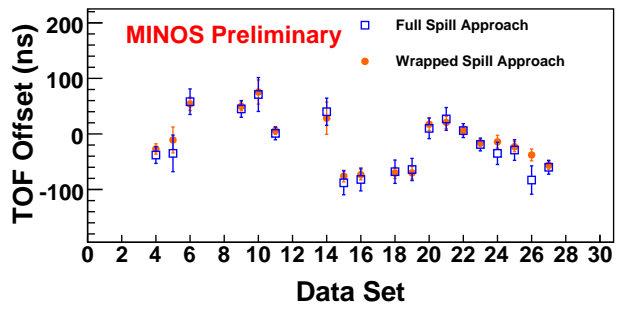

Figure 5: Measured time of flight with respect to the speed of light for the different run periods

statistical error, and with a systematic uncertainty of between 2 and $5 \mathrm{~ns}$, corresponding to a velocity measurement approaching one part in a million. Results from this experiment are expected in late 2012.

$\mathrm{T} 2 \mathrm{~K}$ is in the process of installing a broadly similar system, and could be expected to achieve similar accuracy on the time of flight, and in view of the shorter baseline an uncertainty of more like three parts in a million. It will be in a position to begin to take data in the spring of 2013.

As reported elsewhere at this meeting, OPERA has located and corrected errors in their timing system, and all CNGS experiments now report neutrino velocities consistent with that of light.

\section{Acknowledgments}

Fermilab is operated by Fermi Research Alliance, LLC under contract no. De-AC02-07CH11359 with the United States Department of Energy. The author thanks Thomas Kutter for providing details of the plans of the $\mathrm{T} 2 \mathrm{~K}$ experiment.

\section{References}

[1] G. Kalbfleisch et al., Phys. Rev. Lett. 43, 1361 (1979)

[2] K. Hirata et al., Phys. Rev. Lett. 58, 1490 (1987)

[3] R. M. Bionta et al., Phys. Rev. Lett. 58, 1494 (1987)

[4] E. N. Alekseev et al., JETP Lett. 45, 589 (1987)

[5] M. L. Longo, Phys. Rev. D36, 3276 (1987)

[6] A. G. Cohen \& S. L. Glashow, arXiv:1109.4897 [hep-ex]

[7] P. C. W. Davies \& I. G. Moss, arXiv:1201.3284 [astro-ph.CO]

[8] G. Blewitt et al., Lecture notes in Earth Sciences, Springer Verlag, 30 (1988)

[9] T. Feldmann et al., Proc. 42nd PTTI, November 15-18, 2010 , Reston VA, USA, 509-526

[10] K. Abe et al., Phys. Rev. Lett. 107, 041801, (2011)

[11] T. Adam et al., arXiv:1109.4897v2 [hep-ex]

[12] P. Adamson et al., Phys. Rev. D76, 072005 (2007)

[13] D. G. Michael et al., Nucl. Instrum. Meth. A596, 190228 (2008) 